\title{
AC Conductivity Studies of Polyaniline-polymannuronate Nanocomposites
}

\author{
C. Basavaraja, Na Ri Kim, Eun Ae Jo, R. Pierson, and Do Sung Huh๋
Department of Chemistry and Institute of Functional Material. Inje Universin, Kimhae. Kvungnam 621-749, Korea
E-mail: chemhds ainje ack $\mathrm{kr}$
Received April 25, 2009. Accepted Mav 15, 2009

\begin{abstract}
Temperature and frequency dependent $\mathrm{AC}$ conductivity behavior has been studied for the chemically synthesized polyaniline-polymannuronate (PANI-PM) composites. The temperature $(300-500 \mathrm{~K})$ and frequency $\left(100-10^{\circ} \mathrm{Hz}\right)$ dependent $A C$ conductivity suggests evidence for the transport mechanism in PANI-PM composites. The frequency dependence of $\mathrm{AC}$ conductivity has been investigated by the power law. The frequency exponent ( $s$ ) is determined, and the data suggest that $s$ decreases with temperature. The variation of $s$ with temperature suggests that $\mathrm{AC}$ conduction is due to the correlated barrier hopping.
\end{abstract}

Key Words: Polyaniline. Polymannuronate. Conductivity, Nano-composite, Nanoscopic textures

\section{Introduction}

Intrinsically conducting polymers (ICP). commonly termed as synthetic metals. have been the subject of extensive theoretical and experimental studies. Among ICPs. polyaniline (PANI) has become the subject of special interest due to its relatively low cost. In the last century. PANI has been described in various forms such as aniline black, emeraldine, and nigraniline, which have been prepared either chemically or electrochemically by aniline oxidation. The most common green protonated emeraldine has conductivity on a semiconductor level of the order of $10^{6} \mathrm{~S} \mathrm{~cm}^{-1}$, many orders of magnitude higher than that of common polymers $\left(10^{-9} \mathrm{~S} \mathrm{~cm}^{-1}\right)$ but lower than that of typical metal $\left(>10^{+} \mathrm{S} \mathrm{cm}^{-1}\right)$. The changes in the plysicochemical properties of PANI occurring in response to various external stimuli are used in various applications, ${ }^{1.2}$ such as organic electrodes. sensors, and actuators. ${ }^{3-5}$ Other uses of them are based on the combination of electrical properties of a semiconductor with material characteristics of polymer for the development of "plastic" microelectronics. ${ }^{1,6}$ electro-chromic devices, tailor-made composite system. ${ }^{8,9}$ and "smart" fabrics. ${ }^{1(1)}$ The establishment of the physical properties of PANI reflecting the condition of preparation is thus of fundamental importance. The important property of PANI is that it is soluble directly in organic solvent from the oxidized state. The synthetic method using a dopant with a relatively large molecular weight such as dodecylbenzene sulphonic acid (DBSA). camphor sulphonic acid (CSA), and maphthalene sulphonic acid (NSA) reduces the interchain interaction of PANI chains.

The chemical modification of conducting polymers with carbohydrate polymers could be promising in the preparation of multifunctional conducting materials through oxidativeradical polymerization. The resulting materials will also be interesting in terms of compatibility with environmental and biological systems. In general. incorporation of conducting poly mers such as polyaniline (PANI) into a flexible biopolymer matrix could result in good process ability, with the electrical conduction having the requisite properties like chenical stability toward dopants and solubility under readily accessible conditions. ${ }^{11.12}$ Moreover, materials may show available electrical conductivity and may have good compatibility for hybrid formation with PANI

In our earlier reports. ${ }^{13.14}$ we have studied the synthesis, characterization. and conductivity of PANI-PM composites in detail. The conposites were synthesized using different weight percentages of PM $(5 \% .10 \%, 15 \%$, and $25 \%)$. It has also been suggested that the presence of PM with PANI tends to provide significant interaction that is either direct or indirect through a mechanical association process. The conductivity measurement is an important experimental techuique to probe the microscopic picture of a highly disordered system. The study of the electrical properties of PANI-PM composites becomes necessary both from a fundamental as well as from a technological point of view: Complete information on electrical conduction mechanisms of such polymeric materials should be provided in order to understand the electrical phenomena. In this manuscript. we discuss the electrical properties of PANI-PM contposites. presenting AC conductivity in the temperature range of $300-500 \mathrm{~K}$ and in the frequency range of $10-10^{6} \mathrm{~Hz}$.

\section{Experimental}

The procedure for obtaining PM. the synthesis. the preparation of thin films from the synthesized composites. and the characterization of the powder samples PANI-PM has been described previously. ${ }^{12,14}$ The target mass loading of PM in the conposites varies from 5 to $25 \mathrm{wt} \%$. PANI homo polymer was synthesized under the same conditions for conparison with the composites. The composites are abbreviated as PANI- $y$, where $v$ refers to the wt $\%$ of PM powder used in the polymerization reaction. The $\mathrm{AC}$ measurement was carried out with a Hewlett Packard 4284A Impedance Analyzer within the temperature range of $300-500 \mathrm{~K}$ and frequency range of $100-10^{6} \mathrm{~Hz}$. 


\section{Results and Discussion}

We have studied the frequency-dependent AC conductivity of PANI-PM composites in the frequency range of $100 \cdot 10^{6}$ $\mathrm{Hz}$ and in the temperature range of $300-500 \mathrm{~K}$. Fig. 1 shows the AC conductivity of PANI. PANI-15. and PANI-25 composites at different frequencies. The samples demonstrate frequency-independent behavior in the limit of lower frequencies and a sublinear response at higher frequencies. Dielectric constant and dielectric loss calculated from the $\mathrm{AC}$ conductivity of the composite samples, and the vaniation in dielectric constant and delectric loss for the composites with an increase in temperature at different frequencles are shown in Fig. 2 and 3. respectively. As shown in Fig. 2. there is a variation in dielectric constant of the PANI-PM composites with an increasing temperature at different frequencies. The dielectric constant decreases monotonically with an increasing frequency. The temperature dependence of the dielectric constant in these composites is more pronounced at lower frequencies. $^{15}$

As shown in Fig. 3. the PANI-PM composites show high dielectric loss at a lower frequency. This may be due to the large effective size of the metallic islands in these compounds and the easy charge transfer through well-ordered chains in disordered regions. The dielectric loss with an increase in temperature at different frequency curves for the composites shows that the loss factor increases with an increase in temperature as the frequency is increased. This result is usually associated with ion dnft. dipole polarization. or interfacial polanization. ${ }^{16}$ The increase in dielectric loss with an increasing temperature at a frequency becomes gradually rapid at low temperatures for PANI-5 and forms three different regions for PANI-15. These can be seen between the temperature regions $300-375,375-425$, and $425-500 \mathrm{~K}$. The dielectric loss factor for PANI-25 is a mixed type of PANI-5 and PANI-15. which shows a steady increase from $300-375 \mathrm{~K}$ and later forms another steady region from $375-500 \mathrm{~K}$. The frequencydependent electrical conductivity for a particular temperature

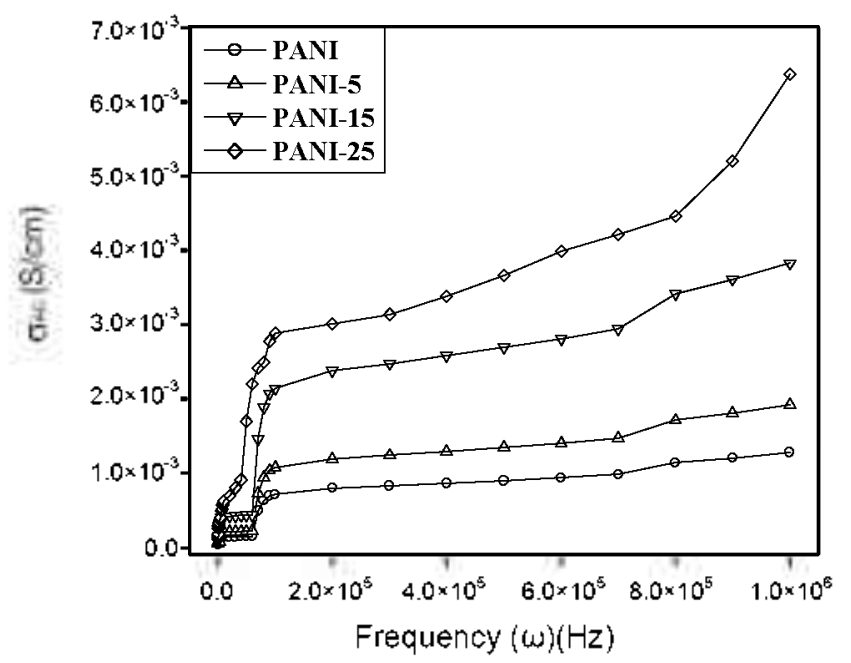

Figure 1. Frequency-dependent AC conductivity of the PANI and PANI-PM composites. and frequency for a disordered senuconductor cau be expressed as ${ }^{1 ? \cdot 19}$

$$
\left.\sigma_{\mathrm{Ac}}(\omega)=\mathrm{A} \omega\right)^{\mathrm{s}}
$$

where $\omega$ is the frequency. $A$ is a constant that depends on $T$,
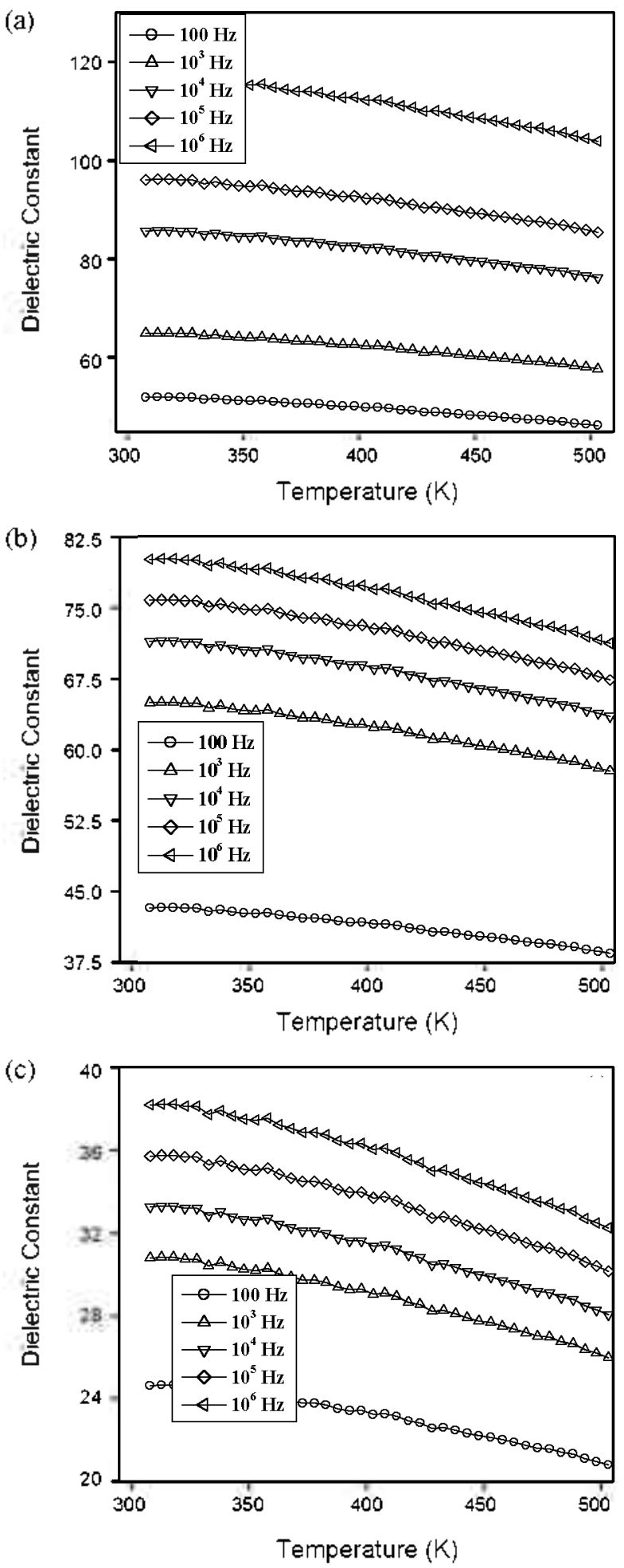

Figure 2. Temperature-dependent dielectnc constant for (a) PANI-5, (b) PANI-15, and (c) PANI-25 composites at different frequencies. 

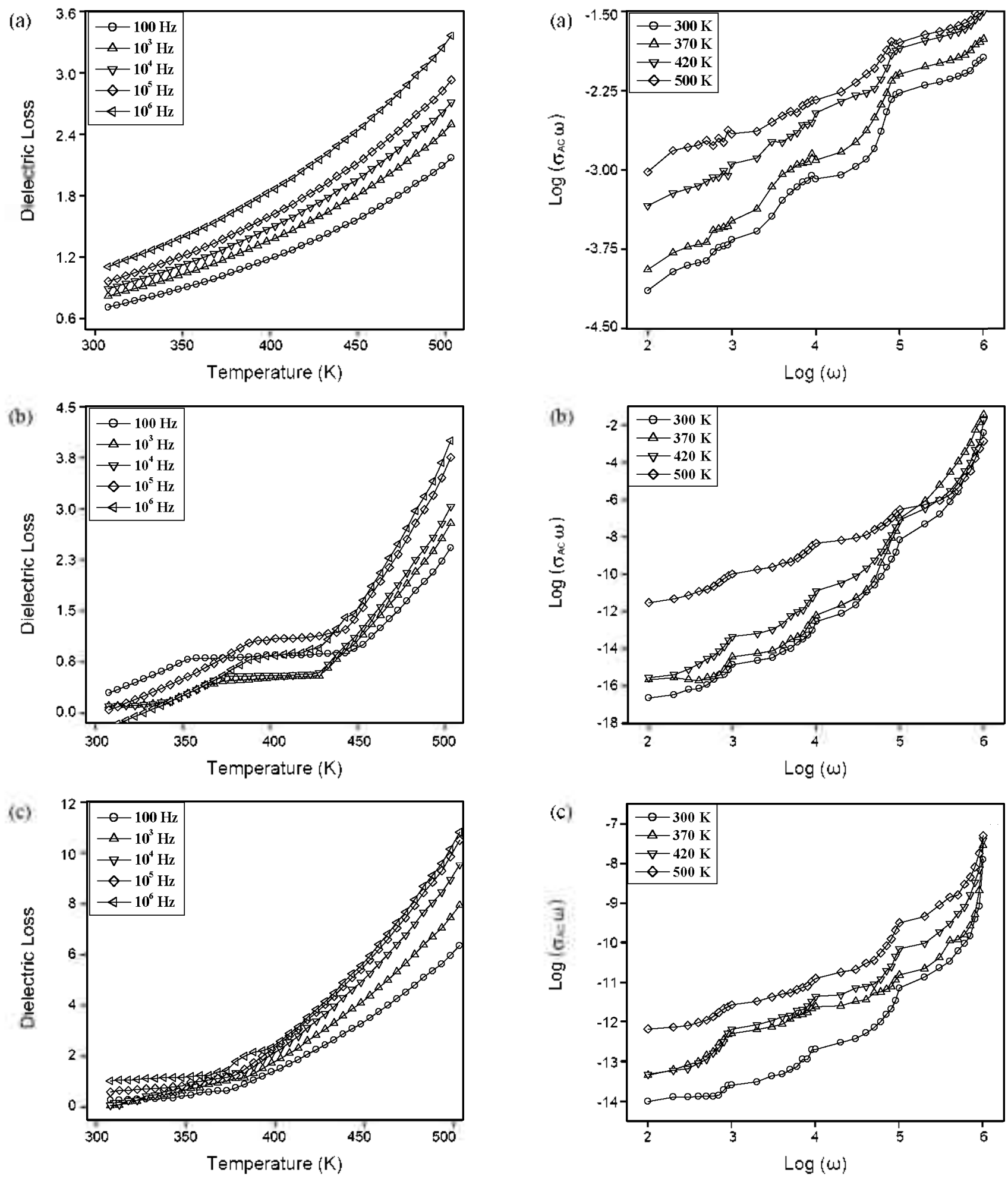

Figure 3. Temperature-dependent Dielectric Loss for (a) PANT-5, (b) PANI-15, and (c) PANI-25 composites at different frequencies.

and the exponent $s \leq 1$. By hopping backward and forward at places with jump probability. a charge carrier may contribute significantly to $\mathrm{AC}$ conductivity. A higher $\omega$ denotes a larger $\mathrm{AC}$ conductivity because better use is made by the place with very large jump probability. ${ }^{20}$ The value of $s$ for different temperatures has been determined from the linear slope of $\log \left(\sigma_{\mathrm{A} C} \omega\right)$ )

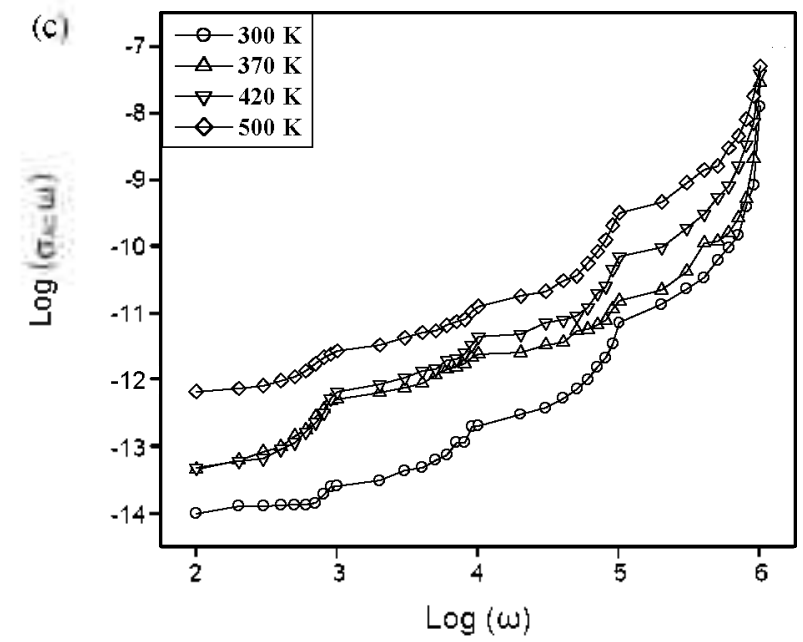

Figure 4. Plot of $\log \left(\sigma_{A C}(0)\right.$ ) $s$. log (o) curves for (a) PANT-5, (b) PANI-15, and (c) PANI-25 composites at different temperatures.

1s. $\log (\omega)$ curves for PANI-5. PANI-15, and PANI-25 composites as shown in Fig. 4. The temperature dependence of $s$ is very complex. Various theoretical models for AC conductivity have been predicted to explain the temperature dependence of $s$.

In this regard. various theoretical nodels for ac conductivity have been predicted to explain the temperature dependence of 


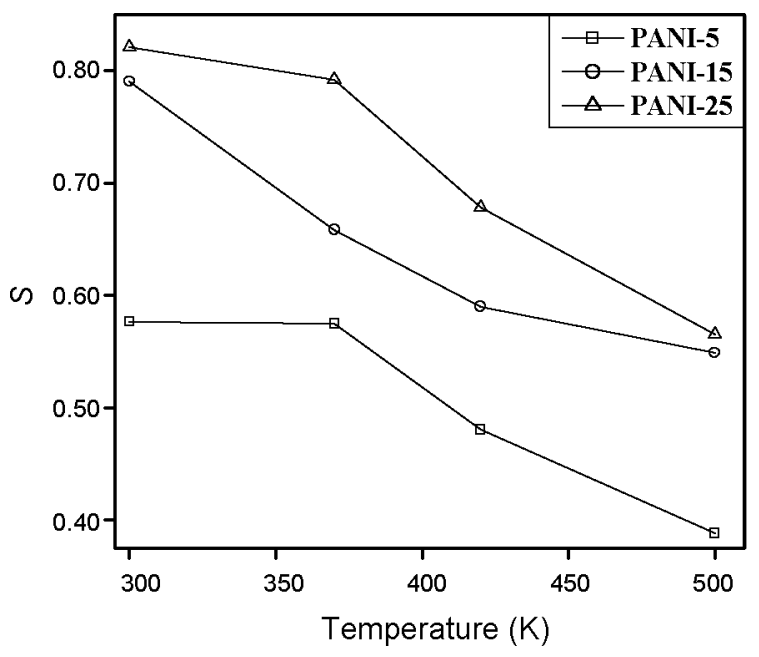

Figure 5. Variation of $s$ with temperature for the composites.

s. The electron turneling model suggests that $s$ is independent of temperature but dependent on frequency ${ }^{-10}$ In the case of small polaron tunneling, $s$ increases, ${ }^{-1}$ whereas for the large polaran tunneling process. $s$ decreases up to a certain temperature and then increases with a further increase in temperature. ${ }^{2-}$ However the value of $s$ of our investigated samples decreases with an increase in temperature. Thus. these models contradict our results. However it is very difficult to explain these facts at this point and requires more data to give a comparative analysis. In the correlated barrier-hopping model $(\mathrm{CBH})^{23}$ the charge carrier hops between the sites over the potential barrier separating them. The frequency exponent $s$ for such model is

$$
s=1-6 k T /\left(H-k T h n\left(1 / l\left(\omega \tau_{1}\right)\right)\right.
$$

where $W$ is the effective barrier height and $\tau_{c}$ is the relaxation time. According to Eq. (2). for the large value of $W k T$. the variation of $s$ with frequency is so small that it is effectively: independent of frequency. ${ }^{34}$ The variation of $s$ with temperature for the PANI-PM composites is shown in Fig. 5. It has been observed in our study that $s$ appears to be independent of frequency $\left[\log \left(\sigma_{A C} \omega\right) v s \cdot \log (\omega)\right.$ curves in Fig. 4]. Therefore. we fitted our experimental data with Eq. (2) as the function of temperature alone with $H^{\circ}$ and $\omega \tau_{i}$ (at a fixed frequency $10^{6}$ $\mathrm{Hz}$ ) lies between $0.015-0.02$ and $3.25 \times 10^{-4}-2.465 \times 10^{-3} \mathrm{~S}$ respectively for different samples. Therefore. from the trend of variation of $s$ with temperature. it may be concluded that AC conductivity in the PANI-PM samples can be described by the $\mathrm{CBH}$ model. Furthermore. the introduction of PM into the PANI matrix will be very usefil. Although several PANI composites exist in the literature, the composite PANI-PM will be very useful in the development of biosensors based on a living system. From our observations and the comparison of PANI-PM with available data on conducting poly mer compo- sites, we suggest that both PM and PANI contribute to the conduction process depending on the volume fraction.

\section{Conclusion}

The chenically synthesized PANI-PM conposites were studied for both temperature and frequency dependent $\mathrm{AC}$ conductivity. It has been observed that the frequency exponent $s$ decreases with the increases in temperature. The dielectric constant decreases monotonically with an increase in frequency. The temperature dependence of the dielectric constant in these composites is more pronounced at lower frequencies. This is because interfacial polarization plays an important role in these composites at lower frequencies. The polarization will arise only when the phases with different conductivities are present. ${ }^{15.24}$ In addition, the property of these composites may be due to the large effective size of the metallic islands in these compounds and the easy charge transfer through wellordered chains in disordered regions.

Aclonowledgments. The authors are grateful for the Korea Research Foundation Grant funded by the Korean Government (MOEHRD) (KRF-00042007070-00).

\section{References}

1. Levi, B. G. Phus. Todon 2000, 53, 19

2. MacDiamid, A. G. Angen: Chent. Int. Ed. 2001, 40,2581

3. Jin, Z.; Su, Y.; Duan, Y. Sens. Actuators B 2001, 72, 75 .

4. Sotomayor, P. T.: Rámurdo, I. M.: Ir, A.; Zarbin, J. G.; Rohwedder, J. J. R; Netto, G. O; Alves, O. L. Sens. Actwators $B$ 2001, 7t, 157 .

5. Kane-Maguire, L. A. P; Wallsce, G. G. Synth. Met 2001, 119, 39.

6. Hamers, R. T. Natuwe 2001, 412,489

7. Rosseinsky, D. R.: Mortimer, R. J. Adh. Mater. 2001, 13, 783.

8. Kim, G. H. ilacromol. Res. 2004, 12, 564 .

9. Cha, S. H.: Kim, I. U.: Lee, T. C. Macromol Res. 2008, 16, 711.

10. El-Sherif, M. E.; Yuan, J-; MacDiarmid, A. G. I. Intell. Mater: Syst. Struct. 2000, 11, 407

11. Mallik, H.; Sarkar, A. J. Non-Civst. Solid. 2006, 352, 795

12. Tiwari, A. J. Poly Res. 2008, 15, 337.

13. Basavaraja, C.; Veranagonda, Y.: Lee, K.: Pierson, R.: Huh, D. S. J. Polv. Sci. B: Polv: Plins. 2009, 47, 36

14. Basavaraja, C.; Veeranagouda, Y.; Lee, K.; Pierson, R.; Huh, D. S. Bull. Korean Chem. Soc. 2008, 29, 1.

15. Prakash, O.; Mandal, K. D; Christopher, C. C. Sastr, M. S.; Kumar, D. J. Matei: Sci. 1996, 31, 4705.

16. Birev, H. J. Appl. Phys. 1978, 19, 2898.

17. Mott, N. F. Davis, E. A. Electronic, Processes I Ron Cnstalline Materials, 2nd Ed: Oxford Clarendon: London, 1979.

18. Long, A. R. Adv Pins. 1982,31,553.

19. Elliott, S. R. Adv Phys. 1987, 36, 135

20. Dyre, J. J. Appl Phys $1988,64,2456$.

21. Long, A. R. Adr Pins. 1982,31,553.

22. Elliott. S. R. Adv Phs $1987,36,135$.

23. Etros A. L. Philos. Hag. B 1981, t3, 829

24. Ghosh, M; Barman, A.: De, S. K.: Chatteriee, S. J.Appl Phys. 1998.84 .806 